\title{
Remdesivir and COVID-19 infection, therapeutic benefits or unnecessary risks?
}

\author{
Antonio Vitiello ${ }^{1}$ (D) $\cdot$ Francesco Ferrara ${ }^{1} \cdot$ Raffaele La Porta $^{2}$ \\ Received: 9 December 2020 / Accepted: 16 December 2020 / Published online: 12 January 2021 \\ (C) Royal Academy of Medicine in Ireland 2021
}

\section{To the editor}

The global COVID-19 pandemic is currently underway. To date, there are about 1.49 million dead and 64.5 million people infected [1]. In this period of global pandemic, several pharmacological treatments have been investigated [2-4]. A good hope to fight COVID-19 comes from the excellent results derived from the latest stages of clinical trials of effective vaccines. However, even when vaccines are available, there will always be a need to continue clinical trials and research to identify effective therapies against SARS-CoV-2. Among all the antiviral drugs used against SARS-CoV-2, remdesivir is currently the subject of scientific debate. Initial evidence has suggested that remdesivir can reduce recovery time for seriously ill patients hospitalized, giving it global attention [5], so much so that emergency use authorization was quickly issued by the US Food and Drug Administration (FDA) in May [6]. Remdesivir represented the therapeutic antiviral treatment against SARS-CoV-2, where the highest hopes were placed during the entire COVID-19 pandemic. However, recently, its role in reducing mortality from COVID-19 seems to be questioned, also considering the recent guidelines developed by the World Health Organization. Indeed, there is conflicting evidence that a treatment cycle of 5 or 10 days leads to clinical improvement. And the World Health Organization's Solidarity study, a huge international study involving thousands of patients, has published provisional results showing that

Antonio Vitiello

antonio.vitiello2@uslumbria1.it

1 Pharmaceutical Department, Usl Umbria 1, XIV Settembre Street, 06121 Perugia, Italy

2 Pathology Department, ASUR Marche, Oberdan 2 Street, 61029 Ancona, Italy the drug does not have a significant impact on mortality or other important outcomes for patients, such as the need for mechanical ventilation or the time needed for clinical improvement [7, 8]. However, some studies, as also reported in other articles [9], associate remdesivir treatment in COVID-19 patients with continuous and clinically significant improvements in positive patients, leading to reduced mortality and reduced recovery time [10-12]. Certainly, therapeutic treatment with remdesivir in COVID-19 patients requires additional data from wellstructured clinical trials. However, it could be considered that the inconsistency of data from some studies is probably due to the specific and subjective immunopathology characteristic of each COVID-19 patient. In addition, if the drug treatment with remdesivir does not bring benefits, its use could still increase the risks of possible adverse reactions; in fact, the information prescribed by the FDA for remdesivir currently includes caution notes on the need to monitor renal and liver function in human patients [13]. Ultimately, what is the current role of remdesivir in the treatment of COVID-19 infection? Some trials have demonstrated its antiviral efficacy in SARS-CoV-2 and faster recovery; others associate lack of efficacy and increased unnecessary risks associated with treatment. Currently, research is focusing on moderately ill patients who could benefit from the drug if administered early. In conclusion, the lack of benefits in the Solidarity study only reinforces the need to better understand the role and effects of remdesivir on the SARS$\mathrm{CoV}-2$ virus.

Authors' contributions I, the undersigned, Antonio Vitiello, and any other author declare that the manuscript was written entirely by the authors. All authors made an equal contribution in the development of the paper.

Antonio Vitiello: conceptualization, writing - original draft, methodology, writing - original draft.

Francesco Ferrara: writing-review and editing, supervision, validation.

Raffaele La Porta: writing-review and editing, supervision, validation. 


\section{Compliance with ethical standards}

The document does not conflict with ethical legislation.

Conflict of interest The authors declare that they have no conflict of interest.

Ethical approval There are no sensitive data, and no patients were recruited for this study. The authors accept the full transfer of copyright to the journal.

\section{References}

1. World Health Organization (WHO) https://www.who.int/ emergencies/diseases/novel-coronavirus-2019/situation-reports (Situation Reports December 2020)

2. Vitiello A, Ferrara F, Pharmacological agents to therapeutic treatment of cardiac injury caused by Covid-19, Life Sciences (Available online 28 September 2020, 118510 ), https://doi.org/ 10.1016/j.lfs.2020.118510

3. Vitiello A, La Porta R, Ferrara F (2020) Sacubitril, valsartan and SARS-CoV-2. BMJ Evid Based Med bmjebm-2020:111497. https://doi.org/10.1136/bmjebm-2020-111497

4. Vitiello A, Ferrara F (2020) Therapeutic strategies for SARS-CoV2 acting on ACE-2. Eur. J. Pharm. Sci. 156:105579. https://doi.org/ 10.1016/j.ejps.2020.105579

5. Wang Y, Zhang D, Du G et al (2020) Remdesivir in adults with severe COVID-19: a randomised, double-blind, placebo-controlled, multicentre trial. Lancet 16(395) 10236:1569-1578

6. US Food and Drug Administration. Coronavirus (Covid-19) update: FDA issues emergency use authorization for potential
COVID-19 treatment. 1 May 2020. www.fda.gov/news-events/ press-announcements/coronavirus-covid-19-update-fda-issuesemergency-use-authorization-potential-covid-19-treatment.

7. WHO Solidarity Trial ConsortiumPan H, Peto R, et al. Repurposed antiviral drugs for covid-19: interim WHO Solidarity trial results. MedRxiv2020.10.15.20209817v1 [Preprint]. 2020. https://doi.org/ 10.1101/2020.10.15.20209817v1. .Google Scholar

8. WHO recommends against the use of remdesivir in COVID-19 patients https://www.who.int/news-room/feature-stories/detail/ who-recommends-against-the-use-of-remdesivir-in-covid-19patients

9. Ferrara F, Porta RL, D'Aiuto V, Vitiello A (2020) Remdesivir and COVID-19. Ir J Med Sci. https://doi.org/10.1007/s11845-02002401-5

10. Vitiello A, Ferrara F (2020 Nov.) Remdesivir versus ritonavir/ lopinavir in COVID-19 patients. Irish Journal of Medical Science. https://doi.org/10.1007/s11845-020-02440-y

11. Sheahan TP, Sims AC, Leist SR, Schäfer A, Won J, Brown AJ, Montgomery SA, Hogg A, Babusis D, Clarke MO, Spahn JE, Bauer L, Sellers S, Porter D, Feng JY, Cihlar T, Jordan R, Denison MR, Baric RS (2020) Comparative therapeutic efficacy of remdesivir and combination lopinavir, ritonavir, and interferon beta against MERS-CoV. Nat. Commun. 11:222-222

12. Beigel JH et al (2020) Remdesivir for the treatment of Covid-19final report. N Engl J Med 8:NEJMoa2007764. https://doi.org/10. 1056/NEJMoa2007764

13. FDA. Prescribing information for Veklury (remdesivir). 2020. www.accessdata.fda.gov/drugsatfda_docs/label/2020/ 214787Orig1s000lbl.pdf.

Publisher's note Springer Nature remains neutral with regard to jurisdictional claims in published maps and institutional affiliations. 